\title{
Joint Value-Distribution of Shifts of the Riemann Zeta-Function
}

\author{
Łukasz Pańkowski@
}

\begin{abstract}
We prove that any complex values $z_{1}, \ldots, z_{n}$ can be approximated by the following integral shifts of the Riemann zeta-function $\zeta(s+$ $\left.i d_{1} \tau\right), \ldots, \zeta\left(s+i d_{n} \tau\right)$ for infinitely many $\tau$, provided $d_{1}, \ldots, d_{n} \in \mathbb{Q}$ are distinct and positive, and $s$ is a fixed complex number lying in the right open half of the critical strip.
\end{abstract}

Mathematics Subject Classification. 11M06.

Keywords. Riemann zeta-function, self-approximation, joint universality, strong reccurence.

\section{Introduction}

In the 80 's of the last century Bagchi (see $[1,2]$ ) observed that the classical Riemann Hypothesis is equivalent to the fact that for every $\varepsilon>0$ and every compact set $K \subset D:=\{s \in \mathbb{C}: 1 / 2<\operatorname{Re}(s)<1\}$ with connected complement we have

$$
\liminf _{T \rightarrow \infty} \frac{1}{T} \text { meas }\left\{\tau \in[0, T]: \max _{s \in K}|\zeta(s+i \tau)-\zeta(s)|<\varepsilon\right\}>0,
$$

where meas denotes the Lebesgue measure on $\mathbb{R}$. In other words (1) means that the set of real $\tau$ satisfying $\max _{s \in K}|\zeta(s+i \tau)-\zeta(s)|<\varepsilon$ has a positive lower density. Moreover, let us note that in the language of topological dynamics Inequality (1) says that the Riemann Hypothesis is equivalent to the strong recurrence of the Riemann zeta-function (see [9]).

The author was partially supported by the grant no. 2016/23/D/ST1/01149 from the National Science Centre. 
One implication in Bagchi's observation is an immediate consequence of the so-called universality theorem due to Voronin [17], which is a generalization of the work of Bohr and his collaborators (see [3-5]) on denseness theorems in $\mathbb{C}$ of values of the Riemann zeta-function and states that for any non-vanishing and continuous function $f(s)$ on $K$, analytic in the interior of $K$, and every $\varepsilon>0$, we have

$$
\liminf _{T \rightarrow \infty} \frac{1}{T} \operatorname{meas}\left\{\tau \in[0, T]: \max _{s \in K}|\zeta(s+i \tau)-f(s)|<\varepsilon\right\}>0 .
$$

The converse implication is a combination of Rouché's theorem and the wellknown zero density theorem for the Riemann zeta-function.

An interesting generalization of (1) was suggested by Nakamura in [11], where he introduced the problem of finding all $d_{1}, d_{2}, \ldots, d_{n}$ such that for every compact set $K \subset D$ with connected complement and every $\varepsilon>0$ we have

$$
\liminf _{T \rightarrow \infty} \frac{1}{T} \operatorname{meas}\left\{\tau \in[0, T]: \max _{1 \leq k \neq \ell \leq n} \max _{s \in K}\left|\zeta\left(s+i d_{k} \tau\right)-\zeta\left(s+i d_{\ell} \tau\right)\right|<\varepsilon\right\}>0 .
$$

Notice that (2) holds for $n=2$ and $d_{1}=0, d_{2} \neq 0$ if and only if the Riemann Hypothesis is true. Interestingly it turns out (see [11-14]) that for $n=2$ the case $d_{1}=0, d_{2} \neq 0$ is the most difficult one as it was shown that (2) holds for $n=2$ and every non-zero real $d_{1}, d_{2}$. A common strategy of showing (2) is to prove a joint version of universality for $\zeta\left(s+i d_{1} \tau\right), \ldots, \zeta\left(s+i d_{n} \tau\right)$, namely to prove that for any non-vanishing and continuous functions $f_{1}(s), \ldots, f_{n}(s)$ on $K$, analytic in the interior of $K$, and every $\varepsilon>0$ we have

$$
\liminf _{T \rightarrow \infty} \frac{1}{T} \text { meas }\left\{\tau \in[0, T]: \max _{1 \leq k \leq n} \max _{s \in K}\left|\zeta\left(s+i d_{k} \tau\right)-f_{k}(s)\right|<\varepsilon\right\}>0 .
$$

Obviously, by taking $f_{1}=f_{2}=\cdots=f_{n}$, one can easily see that (3) implies (2). The problem of finding all parameters $d_{1}, d_{2}$ satisfying (3) for $n=2$ was also completely solved in the aforementioned series of papers by Nakamura and the author, where it was proved that (3) holds for $n=2$ and any real $d_{1}, d_{2}$ with $0 \neq d_{1}, d_{2}$ and $d_{1} \neq \pm d_{2}$. Note that it is indeed a complete answer for $n=2$ as one cannot expect that (3) holds for $d_{1}= \pm d_{2}$.

Unfortunately, the situation for $n \geq 3$ is much more complicated and so far we know only (see [11]) that (3) holds if $d_{1}=1, d_{2}, \ldots, d_{n}$ are algebraic real numbers linearly independent over $\mathbb{Q}$, which is an easier case since for such $d_{k}$ 's, in view of Baker's theorem in transcendental number theory, the set $I\left(d_{1}, \ldots, d_{n}\right):=\left\{d_{k} \log p: p\right.$ is prime, $\left.1 \leq k \leq n\right\}$ is linearly independent over $\mathbb{Q}$ and one can follow straightforwardly the proof of Voronin's theorem in order to get $(3)$. However, if the set $I\left(d_{1}, \ldots, d_{n}\right)$ is not linearly independent over $\mathbb{Q}$, we need a new approach or at least a significant modification of known proofs of universality, since linear independence of the set $I\left(d_{1}, \ldots, d_{n}\right)$ is crucial in several steps of these proofs. 
In the paper we focus on considering the following weaker version of (3) in the flavor of denseness theorems due to Bohr, where $K$ is a singleton and $d_{1}, \ldots, d_{n}$ are positive integers. Note that in this case the set $I\left(d_{1}, \ldots, d_{n}\right)$ is far from being linearly independent over the field of rational numbers.

Theorem 1.1. Let $d_{1}<d_{2}<\cdots<d_{n} \in \mathbb{N}$ and $s=\sigma+i$ with $\sigma \in(1 / 2,1]$ and $t>0$. Then, for every $z_{1}, z_{2}, \ldots, z_{n} \in \mathbb{C}$ and every $\varepsilon>0$ the set of all positive real $\tau$ satisfying

$$
\max _{1 \leq k \leq n}\left|\log \zeta\left(s+i d_{k} \tau\right)-z_{k}\right|<\varepsilon
$$

has a positive lower density.

Corollary 1.2. Let $0<d_{1}<d_{2}<\cdots<d_{n} \in \mathbb{Q}$ and $s=\sigma+$ it with $\sigma \in(1 / 2,1]$ and $t>0$. Then, for every $z_{1}, z_{2}, \ldots, z_{n} \in \mathbb{C}$ and every $\varepsilon>0$ the set of all positive real $\tau$ satisfying

$$
\max _{1 \leq k \leq n}\left|\log \zeta\left(s+i d_{k} \tau\right)-z_{k}\right|<\varepsilon
$$

has a positive lower density.

Proof. Assume that $d_{j}=a_{j} / b_{j}$ with $a_{j}, b_{j} \in \mathbb{N}$. Then it suffices to apply Theorem 1.1 with $L a_{j} / b_{j} \in \mathbb{N}$ instead of $d_{j}$, where $L=\operatorname{lcm}\left(b_{1}, \ldots, b_{n}\right)$, and next make a substitution $L \tau \mapsto \tau$.

Obviously, the above corollary easily implies that (2) holds for $K=\{s\}$ and arbitrary distinct positive rational numbers $d_{1}, \ldots, d_{n}$.

Moreover, it is worth mentioning that the proof of Theorem 1.1 presented in the paper might be simplified in several places under the assumption that $d_{1}, \ldots, d_{n}$ are integers. However, we decided to make all steps in the proof as general as possible, since it might be interesting for the reader to see that in fact the proof of the above theorem is valid for arbitrary positive real $d_{1}, \ldots, d_{n}$ except only one step where we use the implication

$$
-\tau \frac{\log p}{2 \pi} \text { is close to } \theta_{p} \bmod 1 \Longrightarrow-\tau d_{k} \frac{\log p}{2 \pi} \text { is close to } d_{k} \theta_{p} \bmod 1,
$$

which is trivially true for $d_{k} \in \mathbb{Z}$, but it does not necessary hold if $d_{k} \notin \mathbb{Z}$.

\section{Denseness Lemma}

In this section we prove the following denseness lemma, which holds for all positive real $d_{1}, \ldots, d_{n}$.

Lemma 2.1. Let $d_{1}, d_{2}, \ldots, d_{n}$ be real numbers satisfying $0<d_{1}<d_{2}<\cdots<$ $d_{n}$ and $s=\sigma+i t$ with $\sigma \in(1 / 2,1]$ and $t>0$. Then, for every $z_{1}, z_{2}, \ldots, z_{n} \in \mathbb{C}$ 
and every $\varepsilon>0, y>0$, there exist a finite set of prime numbers $M \supset\{p: p<$ $y\}$ and a sequence $\bar{\theta}=\left(\theta_{p}\right)_{p \in M}$ such that

$$
\max _{1 \leq k \leq n}\left|\sum_{p \in M} \frac{e\left(d_{k} \theta_{p}\right)}{p^{s}}-z_{k}\right|<\varepsilon,
$$

where as usual $e(t)=e^{2 \pi i t}$.

The proof of the lemma essentially based on the following Pechersky's [15] generalization of the Riemann rearrangement lemma to Hilbert spaces.

Lemma 2.2. ([15]) Let $H$ be a real Hilbert space with the inner product $(\cdot \mid \cdot)$ and a sequence $\left(u_{n}\right)_{n \in \mathbb{N}} \subset H$ be such that $\sum_{n=1}^{\infty}\left\|u_{n}\right\|_{H}^{2}<\infty$. Moreover, suppose that for every $e \in H$ with $\|e\|=1$ the series $\sum_{n=1}^{\infty}\left\langle u_{n}, e\right\rangle$ are conditionally convergent after suitable permutation of terms. Then, for every $v \in H$ there exists a permutation $\left(n_{k}\right)$ such that $\sum_{k=1}^{\infty} u_{n_{k}}=v$.

Moreover, we use the following effective version of Kronecker approximation theorem due to Chen. Henceforth, $\|\cdot\|$ will denote the distance to the nearest integer.

Lemma 2.3. (Chen, [6, Theorem 1(ii)]) Let $\lambda_{1}, \ldots, \lambda_{n}$ and $\alpha_{1}, \ldots, \alpha_{n}$ be real numbers and assume the following property: for all integers $u_{1}, \ldots, u_{n}$ with $\left|u_{j}\right| \leq M$, the assertion

$$
u_{1} \lambda_{1}+\cdots+u_{n} \lambda_{n} \in \mathbb{Z}
$$

implies that $u_{1} \alpha_{1}+\cdots+u_{n} \alpha_{n}$ is an integer. Then for all positive real numbers $\delta_{1}, \ldots, \delta_{n}$ and for any integers $T_{1}<T_{2}$, we have

$$
\inf _{t \in\left[T_{1}, T_{2}\right] \cap \mathbb{Z}} \sum_{j=1}^{n} \delta_{j}\left\|\lambda_{j} t-\alpha_{j}\right\|^{2} \leq \frac{\Delta}{4} \sin ^{2}\left(\frac{\pi}{2(M+1)}\right)+\frac{\Delta M^{n}}{8\left(T_{2}-T_{1}\right) \Lambda},
$$

where

$$
\Delta=\sum_{j=1}^{n} \delta_{j}
$$

and

$$
\Lambda=\min \left\{\left|u_{1} \lambda_{1}+\cdots+u_{n} \lambda_{n}\right|: u_{j} \in \mathbb{Z},\left|u_{j}\right| \leq M, \sum_{j=1}^{n} \lambda_{j} u_{j} \notin \mathbb{Z}\right\} .
$$

Corollary 2.4. Let $\omega \geq 1, n \in \mathbb{N}$ and $d_{1}, d_{2}, \ldots, d_{n} \in \mathbb{R}$. Then there is an integer $T:=T\left(d_{1}, d_{2}, \ldots, d_{n}, n, \omega\right)>0$ such that for every $a \in \mathbb{Z}$ there exists an integer $h \in[a, a+T]$ satisfying

$$
\sum_{k=1}^{n}\left\|h d_{k}\right\|^{2} \leq \frac{n}{\omega}
$$


Proof. Applying Chen's lemma for $T_{1}=a, T_{2}=a+T,\left(\lambda_{1}, \ldots, \lambda_{n}\right)=$ $\left(d_{1}, d_{2}, \ldots, d_{n}\right), \alpha_{1}=\cdots=\alpha_{n}=0, \delta_{1}=\cdots=\delta_{n}=1$ and $M:=\pi \omega / 4-1$ gives that there is $h \in \mathbb{Z}, h \in[a, a+T]$ such that

$$
\sum_{k=1}^{n}\left\|h d_{k}\right\|^{2} \leq \frac{n}{4}\left(\sin ^{2}\left(\frac{\pi}{2(M+1)}\right)+\frac{M^{n}}{2 \Lambda T}\right) .
$$

Obviously, we have $\sin ^{2}\left(\frac{\pi}{2(M+1)}\right) \leq \frac{2}{\omega}$. Moreover, since $M$ depends only on $\omega$ and $\Lambda$ depends only on $M$ and $d_{1}, \ldots, d_{n}$, there is a sufficiently large $T$ such that $\frac{M^{n}}{2 \Lambda T} \leq \frac{2}{\omega}$, which completes the proof.

The need for the application of Lemma 2.2 forces us to consider the distribution of zeros of functions being linear combinations of exponential functions, which is well understood by the following result due to Wilder.

Lemma 2.5. (Wilder's theorem) Let $g(z)=\sum_{k=1}^{n} A_{k} e^{\omega_{k} z}$, where $z=x+i y \in$ $\mathbb{C}, \mathbb{C} \ni A_{k} \neq 0, k=1,2, \ldots, n$, and $\omega_{1}<\cdots<\omega_{n}$. Then there exsits $K>0$ such that

(i) all zeros of $g(z)$ lie in the strip $|x| \leq K$;

(ii) for each pair $(\alpha, \beta) \in \mathbb{R}^{2}$ with $\beta>0$, we have

$$
\left|N_{0}(\alpha, \beta, K)-\beta \frac{\omega_{n}-\omega_{1}}{2 \pi}\right| \leq n-1,
$$

where $N_{0}(\alpha, \beta, K)$ counts the number of zeros of $g(z)$ in the rectangle $|x| \leq K, y \in[\alpha, \alpha+\beta]$.

Proof. For the proof we refer to [7, Theorem 1].

Proof of Lemma 2.1. Let $\theta_{p_{m}}=\frac{m}{L}$, where $p_{m}$ denotes the $m$-th prime number and $L$ is an arbitrary integer satisfying $L>n-1+d_{n}-d_{1}$. We shall use Lemma 2.2 for the sequence

$$
u_{m}=\left(\frac{e\left(d_{1} \theta_{p_{m}}\right)}{p_{m}^{s}}, \ldots, \frac{e\left(d_{n} \theta_{p_{m}}\right)}{p_{m}^{s}}\right)
$$

and the real Hilbert space $H=\mathbb{C}^{n}$ equipped with the inner product

$$
(\mathbf{x} \mid \mathbf{y})=\operatorname{Re} \sum_{j=1}^{n} x_{j} \overline{y_{j}} \quad \text { for } \mathbf{x}=\left(x_{1}, \ldots, x_{n}\right), \mathbf{y}=\left(y_{1}, \ldots, y_{n}\right) \in \mathbb{C}^{n} .
$$

Obviously, $\sum_{m=1}^{\infty}\left\|u_{m}\right\|_{H}^{2}<\infty$, since $\sigma>1 / 2$. Thus, in order to apply Lemma 2.2 , it suffices to show that for every $e=\left(a_{1}, \ldots, a_{n}\right) \in \mathbb{C}^{n}$ with $\|e\|_{H}=1$ there are two permutations of the series $\sum_{m}\left(u_{m} \mid e\right)$ tending to $+\infty$ and $-\infty$, respectively. In fact, we show only the existence of a permutation of the series, which diverges to $+\infty$, since the case $-\infty$ is similar and can be left to the reader. 
Let us note that

$$
\left(u_{m} \mid e\right)=\operatorname{Re} \sum_{k=1}^{n} \overline{a_{k}} \frac{e\left(d_{k} \theta_{p_{m}}\right)}{p_{m}^{s}}=\frac{1}{p_{m}^{\sigma}} \operatorname{Re}\left(e^{-i t \log p_{m}}\left(\sum_{k=1}^{n} \overline{a_{k}} e\left(d_{k} \theta_{p_{m}}\right)\right)\right)
$$

and consider the function $f(x)=\sum_{k=1}^{n} \overline{a_{k}} e\left(d_{k} x\right), x \in \mathbb{R}$. Putting $A=\sum_{k=1}^{n}$ $\left|a_{k}\right|^{2}$, one can easily deduce from the Cauchy-Schwarz inequality that for every $x \in \mathbb{R}$ we have

$$
\begin{aligned}
|f(x+\tau)-f(x)| & =\left|\sum_{k=1}^{n} \overline{a_{k}} e\left(d_{k} x\right)\left(e\left(d_{k} \tau\right)-1\right)\right| \leq 2 \pi \sum_{k=1}^{n}\left|a_{k}\right|\left\|d_{k} \tau\right\| \\
& \leq 2 \pi \sqrt{A}\left(\sum_{k=1}^{n}\left\|d_{k} \tau\right\|^{2}\right)^{1 / 2} .
\end{aligned}
$$

Now, from Corollary 2.4, we see that there is a positive integer $N$ such that every interval of length $N$ contains an integer $h$ satisfying $\sum_{k=1}^{n}\left\|h d_{k}\right\|^{2} \leq$ $\frac{\varepsilon^{2}}{(2 \pi)^{2} A}$. Therefore, dividing the interval $(0, \infty)$ into disjoint intervals $I_{l}, l \in \mathbb{N}$, of length $N$, one can find integers $\tau_{l} \in I_{l}$ satisfying $\sum_{k=1}^{n}\left\|\tau_{l} d_{k}\right\|^{2} \leq \frac{\varepsilon^{2}}{(2 \pi)^{2} A}$. Hence, for every $x \in \mathbb{R}$ and $l \in \mathbb{N}$, we have

$$
\left|f\left(x+\tau_{l}\right)-f(x)\right| \leq \varepsilon
$$

Now, let us observe that, by Lemma 2.5, the function $f(x)$ has at most $n-1+d_{n}-d_{1}$ zeros in the interval $[0,1]$. Hence, by the choice of $L$, there is $m_{0}$ such that $0 \leq m_{0} \leq L-1$ and $f\left(\frac{m_{0}}{L}\right)=c_{0} \neq 0$. Thus for every $\tau_{l}$ we have

$$
f\left(\frac{m_{0}+L \tau_{l}}{L}\right)=f\left(\frac{m_{0}}{L}+\tau_{l}\right)=c_{0}+\xi_{l}, \quad \text { for suitable }\left|\xi_{l}\right| \leq \varepsilon,
$$

so each interval $\left[m_{0}+k L N, m_{0}+(k+1) N L\right], k=1,2, \ldots$, contains an integer $m_{k}^{\prime}:=m_{0}+L \tau_{l}$ satisfying $f\left(\frac{m_{k}^{\prime}}{L}\right)=c_{0}+\xi_{l}$ with $\left|\xi_{l}\right| \leq \varepsilon$. Assume that $c_{0}=$ $\left|c_{0}\right| e^{i \varphi}$. Then for every $m_{k}^{\prime}$ and $m \in \mathbb{N}$ satisfying

$$
\frac{2 \pi m}{t}-\frac{\pi}{4 t}+\frac{\varphi}{t}<\log p_{m_{k}^{\prime}}<\frac{2 \pi m}{t}+\frac{\pi}{4 t}+\frac{\varphi}{t}
$$

we have $\varphi-t \log p_{m_{k}^{\prime}} \in(-2 \pi m-\pi / 4,-2 \pi m+\pi / 4)$, so $\operatorname{Re}\left(c_{0} \exp \left(-i t \log p_{m_{k}^{\prime}}\right)\right)$ $\geq \frac{c_{0}}{\sqrt{2}}$. Hence, for sufficiently small $\varepsilon>0$, we have

$$
\operatorname{Re}\left(e^{-i t \log p_{m_{k}^{\prime}} f}\left(\frac{m_{k}^{\prime}}{L}\right)\right) \geq \frac{\left|c_{0}\right|}{2}>0
$$


Hence it remains to notice that the fact that $\sigma<1$ and the definition of $m_{k}^{\prime}$ implies that (as usual, $\lfloor x\rfloor$ denotes the greatest integer less than or equal to $x$ )

$$
\begin{aligned}
& \sum_{m_{k}^{\prime}} \frac{1}{p_{m_{k}^{\prime}}^{\sigma}} \\
& \frac{2 \pi m}{t}-\frac{\pi}{4 t}+\frac{\varphi}{t}<\log p_{m_{k}^{\prime}}<\frac{2 \pi m}{t}+\frac{\pi}{4 t}+\frac{\varphi}{t} \\
& \geq \frac{1}{e^{\frac{2 \pi m}{t}+\frac{\pi}{4 t}+\frac{\varphi}{t}}} \sum_{e^{\frac{2 \pi m}{t}-\frac{\pi}{4 t}+\frac{\varphi}{t}}<p_{m_{k}^{\prime}}<e^{\frac{2 \pi m}{t}+\frac{\pi}{4 t}+\frac{\varphi}{t}}} 1
\end{aligned}
$$

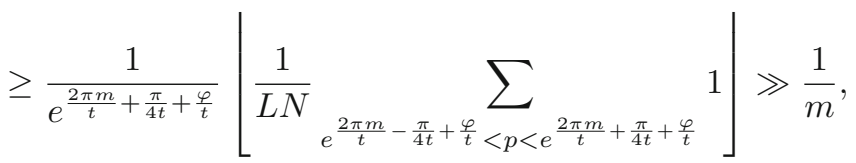

since, for $x=\frac{2 \pi m}{t}+\frac{\varphi}{t}$ and $h=\frac{\pi}{4 t}$, the prime number theorem gives

$$
\begin{aligned}
\sum_{e^{x-h}<p<e^{x+h}} 1 & =\pi\left(e^{x+h}\right)-\pi\left(e^{x-h}\right)+O(1) \\
& =\frac{e^{x+h}}{x+h}-\frac{e^{x-h}}{x-h}+O_{h}\left(\frac{e^{x}}{x^{2}}\right) \\
& \gg_{h} \frac{e^{x}}{x} \gg_{t} \frac{e^{\frac{2 \pi m}{t}+\frac{\pi}{4 t}+\frac{\varphi}{t}}}{m} .
\end{aligned}
$$

Thus using the fact that the harmonic series is divergent completes the proof.

\section{Approximation by a Finite Sum Over Primes}

In this section we use the following Tsang's lemma in order to show that the logarithm of the Riemann zeta-function can be approximated by a truncated Dirichlet series over primes for sufficiently many arguments from any vertical line lying in the right open half of the critical strip.

Lemma 3.1. (Tsang [16, Theorem 5.1 and Remark 1]) Let $\sigma \in(1 / 2,1]$ and $0<\delta<\frac{1}{64}$ be fixed. Then, for sufficiently large $T$, we have

$$
\frac{1}{T} \int_{T}^{2 T}\left|\log \zeta(\sigma+i t)-\sum_{p \leq T^{\delta}} \frac{1}{p^{\sigma+i t}}\right|^{2} d t \ll T^{c(1 / 2-\sigma)},
$$

where $c$ is a positive constant. 
Lemma 3.2. Let $\varepsilon, \varepsilon^{\prime}>0,0<d_{1}<d_{2}<\cdots<d_{n}, s=\sigma+i t$ with $\sigma \in$ $(1 / 2,1], t>0,0<\delta<\frac{1}{65}$ and $C(T, \varepsilon)$ denotes the set of $\tau \in[T, 2 T]$ satisfying

$$
\max _{1 \leq k \leq n}\left|\log \zeta\left(s+i d_{k} \tau\right)-\sum_{p \leq T^{\delta}} \frac{1}{p^{s+i d_{k} \tau}}\right|<\varepsilon .
$$

Then, for sufficiently large $T$, we have

$$
\frac{1}{T} \text { meas } C(T, \varepsilon)>1-\varepsilon^{\prime}
$$

Proof. First let us observe that using Tsang's lemma and a suitable version of Hilbert's inequality due to Montgomery and Vaughan (see [10]) give

$$
\begin{aligned}
& \int_{T}^{2 T}\left|\log \zeta\left(s+i d_{k} \tau\right)-\sum_{p \leq T^{\delta}} \frac{1}{p^{s+i d_{k} \tau}}\right|^{2} d \tau \\
& \ll \int_{d_{k} T+t}^{2\left(d_{k} T+t\right)}\left|\log \zeta(\sigma+i \tau)-\sum_{p \leq T^{\delta}} \frac{1}{p^{\sigma+i \tau}}\right|^{2} d \tau \\
& \ll \int_{d_{k} T+t}^{2\left(d_{k} T+t\right)} \mid \log \zeta(\sigma+i \tau) \\
& \quad-\left.\sum_{p \leq\left(d_{k} T+t\right)^{1 / 65}} \frac{1}{p^{\sigma+i \tau}}\right|^{2} d \tau \\
& \quad+\left.\left.\int_{d_{k} T+t}^{2\left(d_{k} T+t\right)}\right|_{T^{\delta} \leq p \leq\left(d_{k} T+t\right)^{1 / 65}} \frac{1}{p^{\sigma+i \tau}}\right|^{2} d \tau \\
& \ll T^{1+c(1 / 2-\sigma)}+T \sum_{p \leq\left(d_{j} T+t\right)^{1 / 65}} \frac{1}{p^{2 \sigma}} \\
& \ll T^{1+c^{\prime}(1 / 2-\sigma)} \sum^{2}
\end{aligned}
$$

for some $c^{\prime}>0$ and $k=1,2, \ldots, n$. Hence for every $\varepsilon_{0}>0$ and sufficiently large $T$ we have

$$
\sum_{k=1}^{n} \frac{1}{T} \int_{T}^{2 T}\left|\log \zeta\left(s+i d_{k} \tau\right)-\sum_{p \leq T^{\delta}} \frac{1}{p^{s+i d_{k} \tau}}\right|^{2} d \tau<\varepsilon_{0}
$$

Now let

$$
\begin{aligned}
& A_{T}^{\prime}=\left\{\tau \in[T, 2 T]: \sum_{k=1}^{n} \mid \log \zeta\left(s+i d_{k} \tau\right)-\sum_{p \leq T^{\delta}} \frac{1}{\left.p^{s+i d_{k} \tau} \mid>\varepsilon_{0}^{1 / 3}\right\},}\right. \\
& A_{T}^{\prime \prime}=\left\{\tau \in[T, 2 T]: \zeta\left(s+i d_{k} \tau\right)=0 \text { for some } 1 \leq k \leq n\right\} .
\end{aligned}
$$


Obviously, by the holomorphicity of the Riemann zeta-function, we have $\operatorname{meas}\left(A_{T}^{\prime \prime}\right)=0$ for every $T$. Moreover,

$$
\varepsilon_{0}^{2 / 3} \frac{\operatorname{meas}\left(A_{T}^{\prime}\right)}{T}<n^{2} \sum_{k=1}^{n} \frac{1}{T} \int_{A_{T}^{\prime}}\left|\log \zeta\left(s+i d_{k} \tau\right)-\sum_{p \leq T^{\delta}} \frac{1}{p^{s+i d_{k} \tau}}\right|^{2} d \tau \leq n^{2} \varepsilon_{0},
$$

so meas $\left(A_{T}^{\prime}\right) \leq n^{2} \varepsilon_{0}^{1 / 3} T$. Thus, putting $A_{T}=A_{T}^{\prime} \cup A_{T}^{\prime \prime}$ and choosing $\varepsilon_{0}$ sufficiently small, we get that meas $\left(A_{T}\right) \leq \varepsilon^{\prime} T$ and

$$
\sum_{k=1}^{n}\left|\log \zeta\left(s+i d_{k} \tau\right)-\sum_{p \leq T^{\delta}} \frac{1}{p^{s+i d_{k} \tau}}\right|<\varepsilon
$$

for $\tau \in[T, 2 T] \backslash A_{T}$.

\section{Proof of the Main Theorem}

In order to prove our main theorem, we need the following crucial lemma, which allows us to connect Lemmas 2.1 and 3.2. The lemma is essentially Lemma 3.6 from [8] where the quantity $\delta(T)$ defined in Lemma 3.6 in [8] has to be estimated in a more subtle way because of the appearance of an extra parameter $d_{k}$.

Lemma 4.1. Let $d_{1}, d_{2}, \ldots, d_{n}$ be real numbers satisfying $0<d_{1}<d_{2}<\cdots<$ $d_{n}$ and $s=\sigma+i t$ with $\sigma \in(1 / 2,1]$ and $t>0$. Moreover, suppose that $y>0$ is sufficiently large, $0<\delta<\frac{1}{65}, 0<d<1 / 2, M$ is a finite set of prime numbers such that $\{p: p \leq y\} \subset M$ and $\left(\theta_{p}\right)_{p \in M}$ is a sequence of real numbers. Then for sufficiently large $T$ we have

$$
\max _{1 \leq k \leq n} \int_{A_{d}(T)}\left|\sum_{\substack{p \leq T^{\delta} \\ p \notin M}} \frac{1}{p^{s+i d_{k} \tau}}\right|^{2} d \tau \ll T(2 d)^{|M|} y^{1-2 \sigma},
$$

where $A_{d}(T)=\left\{\tau \in[T, 2 T]: \max _{p \in M}\left\|-\tau \frac{\log p}{2 \pi}-\theta_{p}\right\| \leq d\right\}$.

Proof. We will closely follow the proof of Lemma 3.6 from [8].

Let $\delta^{\prime}>0$ be such that $0<d+\delta^{\prime}<1 / 2$ and $\xi: \mathbb{R} \rightarrow[0,1]$ be a continuous and periodic function with period 1 defined on $[-1 / 2,1 / 2]$ by

$$
\xi(x)= \begin{cases}1, & |x| \leq d \\ -\frac{|x|}{\delta^{\prime}}+\frac{d+\delta^{\prime}}{\delta^{\prime}}, & d<|x| \leq d+\delta^{\prime} \\ 0, & d+\delta^{\prime}<|x| \leq \frac{1}{2} .\end{cases}
$$

Moreover, we put

$$
\tilde{\xi}(\tau)=\prod_{p \in M} \xi\left(-\frac{\tau \log p}{2 \pi}-\theta_{p}\right) \quad \text { for } \tau \in \mathbb{R} .
$$


Obviously $\xi(x)=1$ if $\|x\| \leq d$. Hence, for $\tau \in A_{d}(T)$ we have $1=\tilde{\xi}(\tau)=\tilde{\xi}^{2}(\tau)$, whereas for arbitrary $\tau \in \mathbb{R}$ we have $0 \leq \tilde{\xi}^{2}(\tau) \leq \tilde{\xi}(\tau) \leq 1$.

Now let us assume that $\varepsilon_{0}>0$ is small. Then there is a trigonometric polynomial

$$
P(x)=\sum_{-L \leq \ell \leq L} c(\ell) e(\ell x), \quad c(\ell) \in \mathbb{C}
$$

such that $|\xi(x)-P(x)|<\varepsilon_{0}$ for $x \in \mathbb{R}$. So let us define

$$
\tilde{P}(\tau)=\prod_{p \in M} P\left(-\frac{\tau \log p}{2 \pi}-\theta_{p}\right) .
$$

Using the fact that $\left|\prod_{j=1}^{n} a_{j}-\prod_{j=1}^{n} b_{j}\right| \leq R^{n-1} \sum_{j=1}^{n}\left|a_{j}-b_{j}\right|$ for arbitrary complex numbers $a_{1}, \ldots, a_{n}, b_{1}, \ldots, b_{n}$ with $\left|a_{j}\right|,\left|b_{j}\right| \leq R, j=1,2, \ldots, n$, we get

$$
\left|\prod_{p \in M} \xi\left(x_{p}\right)-\prod_{p \in M} P\left(x_{p}\right)\right| \leq 2^{|M|-1}|M| \varepsilon_{0}
$$

for all real $x_{p}$, where $|M|$ denotes the number of elements in the set $M$. Hence $|\tilde{\xi}(\tau)-\tilde{P}(\tau)| \leq 2^{|M|-1}|M| \varepsilon_{0}$ for $\tau \in \mathbb{R}$. Therefore, by the Cauchy-Schwarz inequality, we have

$$
|\tilde{\xi}(\tau)|^{2} \leq 2|\tilde{P}(\tau)|^{2}+2^{2|M|-1}|M|^{2} \varepsilon_{0}^{2}, \quad \tau \in \mathbb{R}
$$

and

$$
|\tilde{P}(\tau)|^{2} \leq 2|\tilde{\xi}(\tau)|^{2}+2^{2|M|-1}|M|^{2} \varepsilon_{0}^{2}, \quad \tau \in \mathbb{R} .
$$

Now, let $N^{\prime}>y$. Then for $\tau \in \mathbb{R}$ we have

$$
\begin{aligned}
\sum_{\substack{p \leq T^{\delta} \\
p \notin M}} \frac{1}{p^{s+i d_{k} \tau}} & =\sum_{\substack{p \leq N^{\prime} \\
p \notin M}} \frac{1}{p^{s}} e\left(-\frac{d_{k} \tau \log p}{2 \pi}\right)+\sum_{\substack{N^{\prime}<p \leq T^{\delta} \\
p \notin \bar{M}}} \frac{1}{p^{s}} e\left(-\frac{d_{k} \tau \log p}{2 \pi}\right) \\
& =: S_{1}(\tau)+S_{2}(\tau),
\end{aligned}
$$

so using the Cauchy-Schwarz inequality again yields

$$
\int_{A_{d}(T)}\left|\sum_{\substack{p \leq T^{\delta} \\ p \notin M}} \frac{1}{p^{s+i d_{k} \tau}}\right|^{2} d \tau \leq 2 \int_{A_{d}(T)}\left|S_{1}(\tau)\right|^{2} d \tau+2 \int_{A_{d}(T)}\left|S_{2}(\tau)\right|^{2} d \tau=: I_{1}+I_{2} .
$$

First let us estimate $I_{1}$. We have

$$
\begin{aligned}
I_{1} \leq & \int_{T}^{2 T}|\tilde{\xi}(\tau)|^{2}\left|S_{1}(\tau)\right|^{2} d \tau \ll \int_{T}^{2 T}|\tilde{P}(\tau)|^{2}\left|S_{1}(\tau)\right|^{2} d \tau \\
& +2^{2|M|-1}|M|^{2} \varepsilon_{0}^{2} \int_{T}^{2 T}\left|S_{1}(\tau)\right|^{2} d \tau
\end{aligned}
$$


and

$$
\begin{aligned}
\tilde{P}(\tau) & =\prod_{p \in M}\left(\sum_{-L \leq \ell_{p} \leq L} c\left(\ell_{p}\right) e\left(\ell_{p}\left(-\frac{\tau \log p}{2 \pi}-\theta_{p}\right)\right)\right) \\
= & \sum_{-L \leq \ell_{p_{1}}, \ldots, \ell_{p_{|M|}} \leq L} \tilde{c}\left(\ell_{p_{1}}, \ldots, \ell_{p_{|M|}}\right) e\left(-\frac{\tau}{2 \pi} \sum_{j=1}^{|M|} \ell_{p_{j}} \log p_{j}\right)
\end{aligned}
$$

where $p_{1}, \ldots, p_{|M|}$ are all elements of $M$ and

$$
\tilde{c}\left(\ell_{p_{1}}, \ldots, \ell_{p_{|M|}}\right)=c\left(\ell_{p_{1}}\right) \cdots c\left(\ell_{p_{|M|}}\right) e\left(-\sum_{j=1}^{|M|} \ell_{p_{j}} \theta_{p_{j}}\right) .
$$

Then

$$
\begin{aligned}
& \int_{T}^{2 T}|\tilde{P}(\tau)|^{2}\left|S_{1}(\tau)\right|^{2} d \tau \\
& \quad=\int_{T}^{2 T} \mid \sum_{-L \leq \ell_{p_{1}}, \ldots, \ell_{p_{|M|}} \leq L} \sum_{\substack{p \leq N^{\prime} \\
p \notin M}} \tilde{c}\left(\ell_{p_{1}}, \ldots, \ell_{p_{|M|}}\right) \frac{1}{p^{s}} e \\
& \quad \times\left.\left(-\frac{\tau}{2 \pi}\left(d_{k} \log p+\sum_{j=1}^{|M|} \ell_{p_{j}} \log p_{j}\right)\right)\right|^{2} d \tau
\end{aligned}
$$

Let

$\Omega_{k}=\left\{-\left(d_{k} \log p+\sum_{j=1}^{|M|} \ell_{p_{j}} \log p_{j}\right): p \leq N^{\prime}, p \notin M,-L \leq \ell_{p_{1}}, \ldots, \ell_{p_{|M|}} \leq L\right\}$

and

$$
\begin{aligned}
& x:=x\left(p, \ell_{p_{1}}, \ldots, \ell_{p_{|M|}}\right)=-\left(d_{k} \log p+\sum_{j=1}^{|M|} \ell_{p_{j}} \log p_{j}\right), \\
& y:=y\left(q, \ell_{p_{1}}^{\prime}, \ldots, \ell_{p_{|M|}}^{\prime}\right)=-\left(d_{k} \log q+\sum_{j=1}^{|M|} \ell_{p_{j}}^{\prime} \log p_{j}\right) .
\end{aligned}
$$

We need to estimate

$$
\delta_{k}=\min _{\substack{x, y \in \Omega_{k} \\\left(p, \ell_{p_{1}}, \ldots, \ell_{p_{|M|}}\right) \neq\left(q, \ell_{p_{1}}^{\prime}, \ldots, \ell_{p_{|M|}}^{\prime}\right)}}|x-y|,
$$

so we consider the following three cases.

Case 1: $p=q$. Then $|x-y|>0$ by the fact that the sequence of logarithms of prime numbers are linearly independent over $\mathbb{Q}$. 
Case 2: $p \neq q, 0 \neq d_{k} \in \mathbb{Q}$. Similarly to the Case 1 , by the unique factorization of integers, we have $x=y \leftrightarrow d_{k}=0, \ell_{p_{i}}^{\prime}=\ell_{p_{i}}^{\prime}$, so $|x-y|>0$. Case 3: $p \neq q, d_{k} \notin \mathbb{Q}$. Then $x=y$ means that for suitable $A, B \in \mathbb{Q}$ we have

$$
d_{k} \log B=d_{k}(\log p-\log q)=\sum_{i=1}^{|M|}\left(\ell_{p_{i}}^{\prime}-\ell_{p_{i}}\right) \log p_{i}=\log A
$$

Using the Six Exponentials Theorem one can prove (see [13, Corollary 2.3 and Lemma 2.4]) the following result.

Lemma 4.2. For arbitrary irrational number $d$ there exist at most two primes $p_{1}, p_{2}$ such that if

$$
d=\frac{\log a}{\log b}, \quad \text { for somea }, b \in \mathbb{Q}
$$

then at least one of $p_{1}, p_{2}$ appears in the prime factorization of $b$.

Now, let us note that $B=p / q$ for some $p, q \notin M$, so $p, q>y$. Hence, taking sufficiently large $y$ and using the above lemma we obtain that (5) never holds, which means that $|x-y|>0$ in Case 3 as well.

Summing up all three cases we get $\delta_{k}>0$. Hence using again a suitable version of Hilbert's inequality due to Montgomery and Vaughan (see [10, Corollary 2, Eq. (1.8)]) we see that for some $\theta_{k}$ with $\left|\theta_{k}\right| \leq 1$ and sufficiently large $T$ we have

$$
\begin{aligned}
& \int_{T}^{2 T}|\tilde{P}(\tau)|^{2}\left|S_{1}(\tau)\right|^{2} d \tau \\
& =\left(T+2 \pi \delta_{k}^{-1} \theta_{k}\right) \sum_{-L \leq \ell_{p_{1}}, \ldots, \ell_{p_{|M|}} \leq L}\left|\tilde{c}\left(\ell_{p_{1}}, \ldots, \ell_{p_{|M|}}\right)\right|^{2} \sum_{\substack{p \leq N^{\prime} \\
p \notin M}} p^{-2 \sigma} \\
& \qquad T y^{1-2 \sigma} \sum_{-L \leq \ell_{p_{1}}, \ldots, \ell_{p_{|M|}} \leq L}\left|\tilde{c}\left(\ell_{p_{1}}, \ldots, \ell_{p_{|M|}}\right)\right|^{2} .
\end{aligned}
$$

Similarly one can obtain

$$
\int_{T}^{2 T}|\tilde{P}(\tau)|^{2} d \tau=\left(T+2 \pi \delta_{0}^{-1} \theta_{0}\right) \sum_{-L \leq \ell_{p_{1}}, \ldots, \ell_{p_{|M|}} \leq L}\left|\tilde{c}\left(\ell_{p_{1}}, \ldots, \ell_{p_{|M|}}\right)\right|^{2}
$$


for some positive real $\delta_{0}$ and $\theta_{0}$ with $\left|\theta_{0}\right| \leq 1$. Thus, we have

$$
\begin{aligned}
T & \sum_{-L \leq \ell_{p_{1}}, \ldots, \ell_{p_{|M|}} \leq L}\left|\tilde{c}\left(\ell_{p_{1}}, \ldots, \ell_{p_{|M|}}\right)\right|^{2} \\
& \ll \int_{T}^{2 T}|\tilde{P}(\tau)|^{2} d \tau \ll \int_{T}^{2 T} \tilde{\xi}(\tau) d \tau+T 2^{2|M|-1}|M|^{2} \varepsilon_{0}^{2} \\
& \leq\left|\int_{T}^{2 T} \tilde{P}(\tau)\right|+T\left(2^{|M|-1}|M| \varepsilon_{0}+2^{2|M|-1}|M|^{2} \varepsilon_{0}^{2}\right) .
\end{aligned}
$$

Note that from (4) we get

$$
\int_{T}^{2 T} \tilde{P}(\tau) d \tau=c(0)^{|M|} T+O(1) .
$$

Moreover, we have

$$
|c(0)|=\left|\int_{-1 / 2}^{1 / 2} P(x) d x\right| \leq \int_{-1 / 2}^{1 / 2}\left(|\xi(x)|+\varepsilon_{0}\right) d x=2 d+\delta^{\prime}+\varepsilon_{0} .
$$

Therefore,

$$
\begin{gathered}
\int_{T}^{2 T}|\tilde{P}(\tau)|^{2}\left|S_{1}(\tau)\right|^{2} d \tau \\
\ll T y^{1-2 \sigma}\left(\left(2 d+\delta^{\prime}+\varepsilon_{0}\right)^{|M|}+2^{|M|-1}|M| \varepsilon_{0}+2^{2|M|-1}|M|^{2} \varepsilon_{0}^{2}\right) .
\end{gathered}
$$

Similarly using Hilbert's inequality one can easily obtain that

$$
\int_{T}^{2 T}\left|S_{1}(\tau)\right|^{2} d \tau \ll T y^{1-2 \sigma}
$$

so

$$
I_{1} \ll T y^{1-2 \sigma}\left(\left(2 d+\delta^{\prime}+\varepsilon_{0}\right)^{|M|}+2^{2|M|+1}|M|^{2} \varepsilon_{0}\right) .
$$

Next, in order to apply Hilbert's inequality to estimate $I_{2}$, we need to use the following estimate

$$
\delta_{k}(T)=d_{k} \min _{\substack{N^{\prime}<p, q \leq T^{\delta} \\ p \neq q \text { and } p, q \notin M}}|\log p-\log q| \geq d_{k} \min _{N^{\prime}<p \leq T^{\delta}} \log \left(1+\frac{1}{p-1}\right) \geq \frac{d_{k}}{T^{\delta}} .
$$

So

$$
I_{2} \leq\left(T+\frac{2 \pi T^{\delta}}{d_{k}}\right) \sum_{\substack{N^{\prime}<p \leq T^{\delta} \\ p \notin \bar{M}}} \frac{1}{p^{2 \sigma}} \ll T N^{1-2 \sigma} .
$$

Choosing $\delta^{\prime}$ and $\varepsilon_{0}$ such that

$$
\left(1+\frac{\delta^{\prime}+\varepsilon_{0}}{2 d}\right)^{|M|}<\frac{3}{2} \quad \text { and } \quad 2^{2|M|+1}|M|^{2} \varepsilon_{0}<\frac{1}{2}(2 d)^{|M|}
$$

implies that $I_{1} \ll T y^{1-2 \sigma}(2 d)^{|M|}$. 
Moreover, if $N^{\prime}$ is such large that $N^{\prime 1-2 \sigma} \leq(2 d)^{|M|} y^{1-2 \sigma}$, then

$$
\int_{A_{d}(T)}\left|\sum_{\substack{p \leq T^{\delta} \\ p \notin M}} \frac{1}{p^{s+i d_{k} \tau}}\right|^{2} d \tau \ll T(2 d)^{|M|} y^{1-2 \sigma},
$$

which completes the proof.

Now, we are in the position to complete the proof of our theorem.

Proof of Theorem 1.1. From Lemma 2.1 we know that there exist a finite set of primes $M \supset\{p: p<y\}$ and a sequence $\left(\theta_{p}\right)_{p \in M}$ of real numbers such that

$$
\max _{1 \leq k \leq n}\left|\sum_{p \in M} \frac{e\left(d_{k} \theta_{p}\right)}{p^{s}}-z_{k}\right|<\varepsilon .
$$

Let $A_{d}(T)=\left\{\tau \in[T, 2 T]: \max _{p \in M}\left\|-\frac{\tau \log p}{2 \pi}-\theta_{p}\right\| \leq d\right\}$ and $d$ is such small that

$$
\max _{1 \leq k \leq n}\left|\sum_{p \in M} \frac{e\left(-d_{k} \frac{\tau \log p}{2 \pi}\right)}{p^{s}}-\sum_{p \in M} \frac{e\left(d_{k} \theta_{p}\right)}{p^{s}}\right|<\varepsilon \quad \text { for any } \tau \in A_{d}(T) .
$$

Let us emphasize that the choice of such $d$ is possible since $d_{1}, \ldots, d_{n} \in \mathbb{N}$, and it is the only step in the proof where we need to assume that $d_{1}, \ldots, d_{n}$ are integers.

Let us notice that for $\tau \in A_{d}(T)$ we have

$$
\max _{1 \leq k \leq n}\left|\sum_{p \in M} \frac{1}{p^{s+i d_{k} \tau}}-z_{k}\right|<2 \varepsilon
$$

Next let

$$
B(T, \varepsilon)=\left\{\tau \in A_{d}(T): \max _{1 \leq k \leq n}\left|\sum_{\substack{p \leq T^{\delta} \\ p \notin M}} \frac{1}{p^{s+i d_{k} \tau}}\right|<\varepsilon\right\},
$$

where $0<\delta<\frac{1}{65}$. Then, by Lemma 4.1 and Kronecker's approximation theorem, for sufficiently large $y$ we have

$$
\frac{1}{T} \operatorname{meas}(B(T, \varepsilon))>\frac{1}{2}(2 d)^{|M|} .
$$

Thus, using the notation from Lemma 3.2, we get that

$$
\max _{1 \leq k \leq n}\left|\log \zeta\left(s+i d_{k} \tau\right)-z_{k}\right|<4 \varepsilon \quad \text { for } \tau \in B(T, \varepsilon) \cap C(T, \varepsilon),
$$


and, since meas $C(T, \varepsilon)>T\left(1-\varepsilon^{\prime}\right)$ for every $\varepsilon^{\prime}>0$, we have

$$
\begin{aligned}
& \frac{1}{T} \text { meas } B(T, \varepsilon) \cap C(T, \varepsilon) \\
& \quad>\frac{1}{T} \text { meas } B(T, \varepsilon)+\frac{1}{T} \text { meas } C(T, \varepsilon)-1>\frac{1}{2}(2 d)^{|M|}-\varepsilon^{\prime}
\end{aligned}
$$

which completes the proof, since the right hand side is positive for sufficiently small $\varepsilon^{\prime}$.

\section{Acknowledgements}

The authors would like to thank the anonymous referee for careful reading of the paper and valuable suggestions.

Funding The author was partially supported by the grant no. 2016/23/D/ST1/ 01149 from the National Science Centre.

Availability of data and material Not applicable.

\section{Declaration}

Conflict of interest The author declares that he has no competing interests.

Code availability Not applicable.

Open Access. This article is licensed under a Creative Commons Attribution 4.0 International License, which permits use, sharing, adaptation, distribution and reproduction in any medium or format, as long as you give appropriate credit to the original author(s) and the source, provide a link to the Creative Commons licence, and indicate if changes were made. The images or other third party material in this article are included in the article's Creative Commons licence, unless indicated otherwise in a credit line to the material. If material is not included in the article's Creative Commons licence and your intended use is not permitted by statutory regulation or exceeds the permitted use, you will need to obtain permission directly from the copyright holder. To view a copy of this licence, visit http://creativecommons. org/licenses/by/4.0/.

\section{References}

[1] Bagchi, B.: The Statistical Behavior and Universality Properties of the Riemann Zeta-Function and Other Allied Dirichlet Series, Ph.D. Thesis, Calcutta, Indian Statistical Institute (1981)

[2] Bagchi, B.: Recurrence in topological dynamics and the Riemann hypothesis. Acta Math. Hung. 50, 227-240 (1987)

[3] Bohr, H.: Zür Theorie der Riemann'schen Zetafunktion im kritischen Streifen. Acta Math. 40, 67-100 (1915) 
[4] Bohr, H., Courant, R.: Neue Anwendungen der Theorie der Diophantischen Approximationen auf die Riemannschen Zetafunktion. J. Reine Angew. Math. 144, 249-274 (1914)

[5] Bohr, H., Jessen, B.: Über die Werteverteilung der Riemannschen Zetafunktion, Erste Mitteilung Acta Math. 54 (1930), 1-35; Zweite Mitteilung, ibid. 58 (1932), $1-55$

[6] Chen, Y.G.: The best quantitative Kronecker's theorem. J. Lond. Math. Soc. (2) 61(3), 691-705 (2000)

[7] Dickson, D.G.: Zeros of exponential sums. Proc. Am. Math. Soc. 16(1), 84-89 (1965)

[8] Gonek, S.M.: Analytic Properties of Zeta and L-Functions, Ph.D. thesis, University of Michigan (1979)

[9] Gottschalk, W.H., Hedlund, G.A., Kulas, M.: Recursive properties of topological transformation groups. Bull. Am. Math. Soc. 52, 488-489 (1946)

[10] Montgomery, H.L., Vaughan, R.C.: Hilbert's inequality. J. Lond. Math. Soc. (2) 8, 73-82 (1974)

[11] Nakamura, T.: The joint universality and the generalized strong recurrence for Dirichlet $L$-functions. Acta Arith. 138, 357-362 (2009)

[12] Nakamura, T., Pańkowski, Ł: Erratum to: the generalized strong recurrence for non-zero rationals parameters. Arch. Math. 99, 43-47 (2012)

[13] Pańkowski, Ł.: Some remarks on the generalized strong recurrence for Lfunctions. In: New Directions in Value Distribution Theory of Zeta and LFunctions, in: Ber. Math., Shaker Verlag, Aachen, pp. 305-315 (2009)

[14] Pańkowski, Ł: Joint universality and generalized strong recurrence for the Riemann zeta function with rational parameter. J. Number Theory 163, 61-74 (2016)

[15] Pecherskii, D.V.: On rearrangements of terms in functional series. Soviet Math. Dokl. 14, 633-636 (1973)

[16] Tsang, K.M.: The Distribution of the Values of the Riemann Zeta-Function, Ph.D. thesis. Princeton University, Princeton (1984)

[17] Voronin, S.M.: Theorem on the universality of the Riemann zeta function, Izv. Akad. Nauk SSSR Ser. Mat. 39: 475-486 (in Russian). Math. USSR Izv. 9(1975), 443-453 (1975)

\section{Łukasz Pańkowski}

Faculty of Mathematics and Computer Science

Adam Mickiewicz University

Uniwersytetu Poznańskiego 4

61-614 Poznań

Poland

e-mail: lpan@amu.edu.pl

Received: December 21, 2020. 
Accepted: January 18, 2022.

Publisher's Note Springer Nature remains neutral with regard to jurisdictional claims in published maps and institutional affiliations. 